\title{
コロケイト入出カの場合の放物型分布定数系に 対する適応レギュレータの設計*
}

\author{
小林 敏弘**.大屋 勝敬**. 樊 家春 $* *$
}

\author{
Adaptive Regulator Design \\ for Collocated Parabolic Distributed Parameter Systems*
}

Toshihiro KOBAYASHI ${ }^{* *}$, Masahiro OYA** and Jiachun FAN**

\begin{abstract}
In this paper an adaptive regulator is designed for collocated parabolic distributed parameter systems with bounded deterministic disturbances in the case of the input and output operators being unbounded. The adaptive regulator is constructed by the concept of high-gain output feedback and the estimation mechanism of the unknown parameters for the disturbance. In the control system, the convergence of the system state to zero will be guaranteed.
\end{abstract}

\section{Introduction}

One of the most important applications of feedback is to achieve regulation and servoaction, that is, to obtain a stable closed-loop system that rejects a given class of external disturbances and tracks a given class of reference signals with zero asymptotic error. This problem has been well investigated provided that the plant is linear and time-invariant and the plant uncertainty is sufficiently small(see Ref. 2) for finite-dimensional systems, and Ref.12) for infinite-dimensional systems).

If the plant uncertainty is large, which is the case if only certain structural information on the plant is available, it is desirable to construct an adaptive regulator or an adaptive servomechanism, that is, a fixed controller that achieves regulation and servoaction for a whole prescribed class of linear time-invariant systems without explicit identification of the system parameters. Nonidentifier-based high-gain adaptive stabilization and adaptive servomechanism design have been also investigated for a class of distributed param-

* 原稿受付 1996 年 5 月 27 日

** 九州工業大学 工学部 Faculty of Engineering, Kyushu Institute of Technology; 1-1 Sensui-cho, Tobata ward, Kitakyushu city, Fukuoka 804, JAPAN

Key Words: adaptive regulator, distributed parameter systems, collocated input and output, boundary input and output. eter systems ${ }^{4), 5), 9), 8), 7), 14)}$. In the design of adaptive control, however, rather strong conditions for distributed parameter systems have been required. These conditions exclude not only the systems with unbounded input and output operators but also the systems with some bounded input and output operators. In Ref. 14) adaptive stabilization has been studied for detectable and stabilizable distributed parameter systems of PrithardSalamon type which are externally stabilized by some scalar output feedback. This class does not allow for collocated actuators and sensors, an interesting case often used in applications. In this paper we shall show that an adaptive regulator can be constructed for a class of collocated parabolic distributed parameter systems with unbounded input and output operators in the case of bounded deterministic disturbances. The adaptive regulator is constructed by the concept of high-gain output feedback and the estimation mechanism of the unknown parameters for the disturbance. In the control system, the convergence of the system state to zero will be guaranteed.

\section{System description and prelimi- nary results}

Consider the distributed parameter system described by the following evolutional equation on a Hilbert space $H$ 


$$
\left\{\begin{aligned}
\frac{d x(t)}{d t}= & A x(t)+B\left[u(t)+\theta^{\mathrm{T}} w(t)\right] \\
& 0<t<t_{f}, x(0)=x_{0} \in H \\
y(t)= & C x(t)
\end{aligned}\right.
$$

where the operator $A$ is the generator of a strongly continuous semigroup on $H$ and $B, C$ are appropriate linear operators. In this paper we only treat the collocated case such that $C=B^{*}\left(B^{*}\right.$ denotes the adjoint of $B$ ). The function $x(t)$ is the system state, $u(t) \in \mathbf{R}$ is the input and $y(t) \in \mathbf{R}$ is the output. The disturbance vector function $w(t)$ is bounded and known, but $\theta$ is the l-dimensional unknown constant vector. For example, we shall consider a disturbance $w(t)$ such that

$$
w(t)=\left[\begin{array}{c}
1 \\
\sin (t) \\
\cos (2 t)
\end{array}\right]
$$

The object of adaptive regulator design is to construct the control input $u$ such that the closedloop system will be stable and reject a given class of disturbances without explicit knowledge of $A$, $B, C$ and $\theta$.

The main conditions required in the design of adaptive stabilizers for the system (1) are the following ${ }^{4), 5), 9)}$

(C1) The $\operatorname{system}(1)$ is minimum-phase, that is, the system has no unstable zeros.

(C2) $C B \neq 0$.

(C3) Range $(\mathrm{B}) \subset$ Domain(A) and Range $\left(\mathrm{C}^{*}\right) \subset$ $\operatorname{Domain}\left(\mathrm{A}^{*}\right)$.

These conditions come from that the inverse system of system (1) is governed by the operator $A_{\gamma}=A-B(C B)^{-1} C(A-\gamma I)$ whose eigenvalues are $\gamma$ and the zeros of system $(1)^{4), 5)}$. The condition $(\mathrm{C} 1)$ is essential in the design of adaptive control systems even for finite-dimensional systems. The condition (C2), in the case of high-gain adaptive stabilization, is the rather major limitation even for finite-dimensional systems. The condition (C3) is proper to distributed parameter systems. This condition excludes some bounded input and output operators such that $B \in L(\mathbf{R}, H)$ (the space of bounded linear operators from $\mathbf{R}$ to $H), C \in$ $L(H, \mathbf{R})$ and any unbounded input and output operators $B, C$. It is a severe restriction from a practical point of view.

In this paper we shall construct a high-gain adaptive regulator for the system (1) in a special case of unbounded input and output operators $B$ and $C=B^{*}$, even if the conditions (C1), (C2) and (C3) do not hold.
Hereafter system description is following Lions $^{6)}$ in order to treat some unbounded input and output operators. Let $H$ and $V$ be two Hilbert spaces with $V, H$ and $V^{\prime}$ (the dual space of $V$ )satisfying the inclusion relation

$$
V \subset H=H^{\prime} \subset V^{\prime}
$$

where $\subset$ means continuous dense injection. We denotes by $\langle\cdot, \cdot\rangle_{X}$ and $\|\cdot\|_{X}$ the scalar product in $X$ and the norm on $X$, respectively. If $f \in V^{\prime}$ and $v \in V,\langle f, v\rangle$ denotes their scalar product. If $f \in H$ , it coincides with the scalar product in $H$. Let the operator $A$ be in $L\left(V, V^{\prime}\right)$ and satisfy the coercivity condition

$$
\operatorname{Re}\langle-A x, x\rangle+c\|x\|_{H}^{2} \geq \delta\|x\|_{V}^{2}, \delta>0, x \in V .
$$

Then the operator $A$ generates a strongly continuous semigroup $S(t)$ on $H$.

For the operators $B$ and $C$ we assume that $B \in L\left(\mathbf{R}, V^{\prime}\right)$ and $C=B^{*} \in L(V, \mathbf{R})$. These $B$ and $C$ can express boundary or pointwise controls and observation. They are called unbounded input and output operators, because Range $(B)$ and $\operatorname{Domain}(C)$ do not coincide with the state space $H$. Then $C B$ is not well-defined and the condition (C3) dose not hold.

For $u \in L^{2}\left(0, t_{f}\right)$ the system (1) has unique solution $x$ such that $x \in L^{2}\left(0, t_{f} ; V\right)$ and $\dot{x} \in$ $L^{2}\left(0, t_{f} ; V^{\prime}\right)^{6)}$. Here $L^{2}\left(0, t_{f} ; X\right)$ denotes the space(equivalent class) of square integrable functions on $\left[0, t_{f}\right]$ with values in $X$.

In this paper we shall show that a high-gain adaptive regulator can be designed for the system (1) with collocated actuators and sensors(that is, $\left.C=B^{*}\right)$ under the following assumptions.

【Assumption 1】 The operator $-A$ is selfadjoint and coercive with compact resolvent, and

$$
C=B^{*} \in L(V, \mathbf{R}) .
$$

【Assumption 2】 There exists a positive constant $\hat{k}$ such that the operator

$$
A_{\hat{k}}=A-\hat{k} B B^{*}
$$

generates an exponentially stable semigroup on $H$.

【Remark 1】 Assumption 2 does not require that the system is minimum-phase.

\section{Adaptive regulators}

In this section, under Assumption $\mathbf{1}$ and Assumption 2, we shall design an adaptive regulator for the system (1) by the concept of high-gain 
output feedback and the estimation mechanism of the unknown parameters for the disturbance. If the parameters $\hat{k}$ and $\theta$ are known, using the feedback control law

$$
u(t)=-\hat{k} y(t)-\theta^{\mathrm{T}} w(t)
$$

for the system (1), we have the closed-loop system

$$
\frac{d x(t)}{d t}=\left(A-\hat{k} B B^{*}\right) x(t) .
$$

Thus a regulator is constructed under Assumption 1 and Assumption 2. We shall show that, even if the parameters $\hat{k}$ and $\theta$ are unknown, an adaptive controller

$$
\left\{\begin{array}{l}
u(t)=-k(t) y(t)-\hat{\theta}(t)^{\mathrm{T}} w(t) \\
\dot{k}(t)=r y^{2}(t), k(0)=0, r>0 \\
\dot{\hat{\theta}}(t)=\Gamma w(t) y(t), \hat{\theta}(0)=\hat{\theta}_{0}
\end{array}\right.
$$

(where $\Gamma$ is an $l$-dimensional positive definite matrix) will regulate the system (1) under Assumption 1 and Assumption 2.

If the-adaptive controller (6) is applied to the system (1), the resulting closed-loop system becomes

$$
\begin{aligned}
& \frac{d x(t)}{d t}=\left[A-k(t) B B^{*}\right] x(t) \\
& -B\left[\hat{\theta}(t)^{\mathrm{T}}-\theta^{\mathrm{T}}\right] w(t) \\
& \left\{\begin{array}{l}
\dot{k}=r y^{2}(t), k(0)=0 \\
\dot{\hat{\theta}}(t)=\Gamma w(t) y(t), \hat{\theta}(0)=\hat{\theta}_{0} \\
y(t)=B^{*} x(t)
\end{array}\right.
\end{aligned}
$$

In this time-variant system (7), the operator $-A+k(t) B B^{*} \in L\left(V, V^{\prime}\right)$ satisfies the coercivity condition for $k(t)<\infty$. When $k(t)<\infty$ and $\|\hat{\theta}(t)\|<\infty$ for $t>0$, the system (7) also has a unique solution $x$ such that $x \in L^{2}\left(0, t_{f} ; V\right)$ and $\dot{x} \in L^{2}\left(0, t_{f} ; V^{\prime}\right)$ for any $t_{f}<\infty^{6)}$. Under Assumption 1 and Assumption 2. the following main theorem holds.

【Theorem 1】 Suppose that Assumption 1 and Assumption 2 hold. Then the resulting closed-loop system (7) and (8) has following properties. For all $x_{0} \in H$ and $k(0)=0$, the solution of eqs.(7) and (8) satisfies

$$
\lim _{t \rightarrow \infty}\|x(t)\|_{H}=0
$$

and

$$
k(t)<\infty \text { and }\|\hat{\theta}(t)\|<\infty \text { for any } t>0
$$

(Proof) The system (7) and (8) can be rewritten as

$$
\left\{\begin{array}{l}
\frac{d x(t)}{d t}=\left(A-\hat{k} B B^{*}\right) x(t)-[k(t)-\hat{k}] B B^{*} x(t) \\
\quad-B\left[\hat{\theta}(t)^{\mathrm{T}}-\theta^{\mathrm{T}}\right] w(t) \\
\dot{k}=r y^{2}(t), k(0)=0 \\
\dot{\hat{\theta}}(t)=\Gamma w(t) y(t), \hat{\theta}(0)=\hat{\theta}_{0} \\
y(t)=B^{*} x(t)
\end{array}\right.
$$

From Assumption 1, $A-k B B^{*} \in L\left(V, V^{\prime}\right.$ is self-adjoint with compact resolvent ${ }^{11)}$, so the spectrum of $A-k B B^{*}$ is discrete and has orthonormal eigenfunctions $\phi_{k n}$ with corresponding eigenvalues $\lambda_{k n}$. The eigenvalues depend continuously on parameter $k^{11)}$. Moreover from Assumption 2 it holds that

$$
\begin{aligned}
\left\langle\left(A-\hat{k} B B^{*}\right) x, x\right\rangle= & \sum_{n=1}^{\infty} \lambda_{\hat{k} n}\left\langle x, \phi_{\hat{k} n}\right\rangle_{H}^{2} \\
& \leq \max _{n} \lambda_{\hat{k} n}\|x\|_{H}^{2}
\end{aligned}
$$

for $x \in V$ and $-\omega_{\hat{k}}=\max _{n} \lambda_{\hat{k} n}<0$.

Now consider a Lyapunov-like functional on $V \times \mathbf{R} \times \mathbf{R}^{l}$

$$
\begin{aligned}
V(t)= & \|x(t)\|_{H}^{2}+\frac{1}{r}[k(t)-\hat{k}]^{2} \\
& +[\hat{\theta}(t)-\theta]^{\mathrm{T}} \Gamma^{-1}[\hat{\theta}(t)-\theta] .
\end{aligned}
$$

The time derivative of $V(t)$ along the solution of system (11) can be estimated as follows.

$$
\begin{aligned}
\dot{V}= & 2\left\langle\left(A-\hat{k} B B^{*}\right) x(t), x(t)\right\rangle \\
& -2[k(t)-\hat{k}]\left\langle B B^{*} x(t), x(t)\right\rangle \\
& +2[k(t)-\hat{k}] y^{2}(t) \\
& -2\left\langle B[\hat{\theta}(t)-\theta]^{\mathrm{T}} w(t), x(t)\right\rangle \\
& +2[\hat{\theta}(t)-\theta]^{\mathrm{T}} w(t) y(t) \\
= & 2\left\langle\left(A-\hat{k} B B^{*}\right) x(t), x(t)\right\rangle \\
& \leq-2 \omega_{\hat{k}}\|x(t)\|_{H}^{2}
\end{aligned}
$$

From this $V(t) \leq V(0)$, and $k(t)<\infty$ and $\|\hat{\theta}(t)\|<$ $\infty$ for any $t>0$ which implies that $y \in L^{2}(0, \infty)$. Since the operator $A_{\hat{k}}=A-\hat{k} B B^{*}$ is self-adjoint and negative-definite, it generates an exponentially stable analytic semigroup $S_{\hat{k}}(t)$ on $H$ such that

$$
\left\|S_{\hat{k}}(t) x\right\|_{H} \leq M e^{-\gamma t}\|x\|_{H} \text { for any } t>0
$$

where $M$ is a positive constant and $0<\gamma \leq \omega_{\hat{k}}^{10)}$ 
We can define $\sqrt{-A_{\hat{k}}}$ and $\left(\sqrt{-A_{\hat{k}}}\right)^{-1}$ in the usual way. Moreover it holds that the domain of $\sqrt{-A_{\hat{k}}}$ is $\mathrm{V}($ Ref. 13), p. 29). We shall estimate the solution $x(t)$ of the system (9). At first, since from eq.(14)

$$
\begin{aligned}
& \|x(t)\|_{H}^{2} \leq-\frac{1}{2 \omega_{\hat{k}}} \frac{d V(t)}{d t}, \\
& \int_{0}^{\infty}\|x(t)\|_{H}^{2} d t \leq \frac{1}{2 \omega_{\hat{k}}}[V(0)-V(\infty)]<\infty .
\end{aligned}
$$

In order to investigate the convergence of $x(t)$ in $H$ we use the eigenvalues $\lambda_{\hat{k} n}$ and the eigenfunctions $\phi_{\hat{k} n}$ of the operator $A-\hat{k} B B^{*}$. In this case we can estimate $x(t)$ as follows. The solution $x(t)$ of the system (7) is given by

$$
\begin{aligned}
x(t)= & S_{\hat{k}}(t) x_{0}-\int_{0}^{t} S_{\hat{k}}(t-\tau)[k(\tau)-\hat{k}] B y(\tau) d \tau \\
& -\int_{0}^{t} S_{\hat{k}}(t-\tau) B[\hat{\theta}(\tau)-\theta]^{\mathrm{T}} w(\tau) d \tau . \quad(17)
\end{aligned}
$$

Here we decompose $x(t)$ such that

$$
\left\{\begin{array}{l}
x(t)=x_{1}(t)+x_{2}(t) \\
x_{1}(t)=S_{\hat{k}}(t) x_{0}+\int_{0}^{t} S_{\hat{k}}(t-\tau) B g_{1}(\tau) d \tau \\
x_{2}(t)=\int_{0}^{t} S_{\hat{k}}(t-\tau) B g_{2}(\tau) d \tau
\end{array}\right.
$$

where $g_{1}(t)=-[k(t)-\hat{k}] y(t)$ and $g_{2}(t)=-[\hat{\theta}(t)-$ $\theta]^{\mathrm{T}} w(t)$. It should be noted that $x_{1}(t)$ and $x_{2}(t)$ are the solutions of the evolution equations

$$
\begin{aligned}
\dot{x}_{1}(t)= & \left(A-\hat{k} B B^{*}\right) x_{1}(t) \\
& +B g_{1}(t), x_{1}(0)=x_{0},
\end{aligned}
$$

and

$$
\begin{aligned}
\dot{x}_{2}(t)= & \left(A-\hat{k} B B^{*}\right) x_{2}(t) \\
& +B g_{2}(t), \quad x_{2}(0)=0,
\end{aligned}
$$

respectively.

First we shall estimate $x_{1}(t)$. From eq.(15)

$$
\left\|S_{\hat{k}}(t) x_{0}\right\|_{H} \leq M e^{-\gamma t}\left\|x_{0}\right\|_{H} .
$$

For the second term we can estimate the function $a(t)$

$$
a(t)=\int_{0}^{t} S_{\hat{k}}(t-\tau) B g_{1}(\tau) d \tau
$$

in $H$ as follows. We can have

$$
\begin{aligned}
& \left|\left\langle a(t), \phi_{\hat{k} n}\right\rangle_{H}\right| \\
& =\left|\left\langle\int_{0}^{t} S_{\hat{k}}(t-\tau)[k(\tau)-\hat{k}] B y(\tau) d \tau, \phi_{\hat{k} n}\right\rangle_{H}\right| \\
& \leq \sup _{t \geq 0}\left|k(t)-\hat{k} \| B^{*} \phi_{\hat{k} n}\right| \int_{0}^{t} e^{\lambda_{\hat{k} n}(t-\tau)}|y(\tau)| d \tau \\
& =K\left|B^{*} \phi_{\hat{k} n}\right|\left(\int_{0}^{\frac{t}{2}} e^{\lambda_{\hat{k} n}(t-\tau)}|y(\tau)| d \tau\right. \\
& \left.+\int_{\frac{t}{2}}^{t} e^{\lambda_{\hat{k} n}(t-\tau)}|y(\tau)| d \tau\right) \\
& =K\left|B^{*} \phi_{\hat{k} n}\right|\left(\int_{\frac{t}{2}}^{t} e^{\lambda_{\hat{k} n} \tau}|y(t-\tau)| d \tau\right. \\
& \left.+\int_{\frac{t}{2}}^{t} e^{\lambda_{\hat{k} n}(t-\tau)}|y(\tau)| d \tau\right) \\
& \leq K\left|B^{*} \phi_{\hat{k} n}\right| \\
& \left\{\left(\int_{\frac{t}{2}}^{t} e^{2 \lambda_{\hat{k} n} \tau} d \tau\right)^{\frac{1}{2}}\left(\int_{\frac{t}{2}}^{t} y^{2}(t-\tau) d \tau\right)^{\frac{1}{2}}\right. \\
& \left.+\left(\int_{\frac{t}{2}}^{t} e^{2 \lambda_{\hat{k} n}(t-\tau)} d \tau\right)^{\frac{1}{2}}\left(\int_{\frac{t}{2}}^{t} y^{2}(\tau) d \tau\right)^{\frac{1}{2}}\right\} \\
& \leq K\left|B^{*} \phi_{\hat{k} n}\right| \\
& \left\{\left[\frac{\exp \left(2 \lambda_{\hat{k} n} t\right)-\exp \left(\lambda_{\hat{k} n} t\right)}{2 \lambda_{\hat{k} n}}\right]^{\frac{1}{2}}\right. \\
& \times\left(\int_{0}^{\infty} y^{2}(\tau) d \tau\right)^{\frac{1}{2}} \\
& \left.+\left[\frac{\exp \left(\lambda_{\hat{k} n} t\right)-1}{2 \lambda_{\hat{k} n}}\right]^{\frac{1}{2}}\left(\int_{\frac{t}{2}}^{\infty} y^{2}(\tau) d \tau\right)^{\frac{1}{2}}\right\} \\
& \leq K\left|B^{*} \phi_{\hat{k} n}\right| \\
& \left\{\left[\frac{-\exp \left(\lambda_{\hat{k} n} t\right)}{2 \lambda_{\hat{k} n}}\right]^{\frac{1}{2}}\left(\int_{0}^{\infty} y^{2}(\tau) d \tau\right)^{\frac{1}{2}}\right. \\
& \left.+\left[\frac{-1}{2 \lambda_{\hat{k} n}}\right]^{\frac{1}{2}}\left(\int_{\frac{t}{2}}^{\infty} y^{2}(\tau) d \tau\right)^{\frac{1}{2}}\right\}
\end{aligned}
$$

From this estimate we can obtain

$$
\begin{aligned}
\|a(t)\|_{H}^{2}= & \sum_{n=1}^{\infty}\left|\left\langle a(t), \phi_{\hat{k} n}\right\rangle_{H}\right|^{2} \\
\leq & 2 K^{2} \sum_{n=1}^{\infty}\left\{\frac{-\exp \left(\lambda_{\hat{k} n} t\right)}{2 \lambda_{\hat{k} n}} \int_{0}^{\infty} y^{2}(\tau) d \tau\right. \\
& \left.+\left[\frac{-1}{2 \lambda_{\hat{k} n}}\right]\left(\int_{\frac{t}{2}}^{\infty} y^{2}(\tau) d \tau\right)\right\}\left|B^{*} \phi_{\hat{k} n}\right|^{2} \\
\leq & K^{2}\left\{e^{\max \lambda_{\hat{k} n} t} \int_{0}^{\infty} y^{2}(\tau) d \tau\right. \\
& \left.+\int_{\frac{t}{2}}^{\infty} y^{2}(\tau) d \tau\right\} \\
& \sum_{n=1}^{\infty} \frac{1}{\left|\lambda_{\hat{k} n}\right|}\left|B^{*} \phi_{\hat{k} n}\right|^{2}<\infty
\end{aligned}
$$

since the domain of $\sqrt{-A_{\hat{k}}}$ is $\mathrm{V}$ and $B \in L\left(\mathbf{R}, V^{\prime}\right)$. Eq.(20) implies that $a(t)$ in $H$ for all $t>0$ and $\|a(t)\|_{H} \rightarrow 0$ as $t \rightarrow \infty$, since $\int_{t}^{\infty} y^{2}(\tau) d \tau \rightarrow 0$ as $t \rightarrow \infty$, if $y \in L^{2}(0, \infty)$. From eqs.(19) and 
(20) it holds that $\left\|x_{1}(t)\right\|_{H}<\infty$ for any $t>0$ and $\lim _{t \rightarrow \infty}\left\|x_{1}(t)\right\|_{H}=0$.

Moreover estimating directly $S_{\hat{k}}(t) x_{0}$ and $a(t)$, we can obtain

$$
\int_{0}^{\infty}\left\|x_{1}(t)\right\|_{H}^{2} d t<\infty .
$$

Next we shall estimate $x_{2}(t)$. Consider the functional

$$
f(t)=\frac{1}{2}\left\|x_{2}(t)\right\|_{H}^{2} .
$$

From eqs.(16) and (21)

$$
\begin{aligned}
\int_{0}^{\infty} f(t) d t \leq & \int_{0}^{\infty}\|x(t)\|_{H}^{2} d t \\
& +\int_{0}^{\infty}\left\|x_{1}(t)\right\|_{H}^{2} d t<\infty
\end{aligned}
$$

that is, $f \in L^{1}(0, \infty)$. On the other hand it holds that

$$
\begin{aligned}
\frac{d f(t)}{d t} & =\left\langle\dot{x}_{2}(t), x_{2}(t)\right\rangle \\
& =\left\langle\left(\sqrt{-A_{\hat{k}}}\right)^{-1} \dot{x}_{2}(t), \sqrt{-A_{\hat{k}}} x_{2}(t)\right\rangle_{H} \\
& \leq\left\|\sqrt{-A_{\hat{k}}} x_{2}(t)\right\|_{H} \cdot\left\|\left(\sqrt{-A_{\hat{k}}}\right)^{-1} \dot{x}_{2}(t)\right\|_{H} \\
& <\infty \text { for any } \quad t>0
\end{aligned}
$$

from Lemma 1(Lemma 1 will be given below). The function $f(t)$ is uniformly continuous for $t>0$ and $f \in L^{1}(0, \infty)$. Hence $\lim _{t \rightarrow \infty} f(t)=0$, that is, $\lim _{t \rightarrow \infty}\left\|x_{2}(t)\right\|_{H}=0$. Consequently we have that $\lim _{t \rightarrow \infty}\|x(t)\|_{H}=0$. We have proved the theorem.

Is should be noted that the exponential convergence of $x(t)$ has not been shown ${ }^{3)}$

Lastly we must prove the following Lemma.

【Lemma 1】 Suppose that the assumptions of Theorem 1 hold. Then

$$
\left\|\sqrt{-A_{\hat{k}}} x_{2}(t)\right\|_{H}<\infty \text { for any } t>0
$$

and

$$
\left\|\left(\sqrt{-A_{\hat{k}}}\right)^{-1} \dot{x}_{2}(t)\right\|_{H}<\infty \text { for any } t>0 .
$$

(Proof) First we can estimate the function $b(t)=\sqrt{-A_{\hat{k}}} x_{2}(t)$.

$$
\begin{aligned}
b(t) & =\sqrt{-A_{\hat{k}}} x_{2}(t) \\
& =\sqrt{-A_{\hat{k}}} \int_{0}^{t} S_{\hat{k}}(t-\tau) B g_{2}(\tau) d \tau
\end{aligned}
$$

in $H$ as follows.

$$
\begin{aligned}
\left|\left\langle b(t), \phi_{\hat{k} n}\right\rangle_{H}\right| & \\
= & \left|\left\langle\int_{0}^{t} \sqrt{-A_{\hat{k}}} S_{\hat{k}}(t-\tau) B g_{2}(\tau) d \tau, \phi_{\hat{k} n}\right\rangle_{H}\right| \\
\leq & \sup _{t \geq 0}\left|[\hat{\theta}(t)-\theta]^{\mathrm{T}} w(t)\right|\left|B^{*} \phi_{\hat{k} n}\right| \\
& \sqrt{-\lambda_{\hat{k} n}} \int_{0}^{t} e^{\lambda_{\hat{k} n}(t-\tau)} d \tau \\
\leq & M_{\theta}\left|B^{*} \phi_{\hat{k} n}\right| \frac{1}{\sqrt{-\lambda_{\hat{k} n}}},
\end{aligned}
$$

where $M_{\theta}=\sup _{t \geq 0} \mid\left[\hat{\theta}(t)-\theta^{\mathrm{T}} w(t) \mid<\infty\right.$. From this we obtain

$$
\begin{aligned}
\|b(t)\|_{H}^{2} & =\sum_{n=1}^{\infty}\left|\left\langle b(t), \phi_{\hat{k} n}\right\rangle_{H}\right|^{2} \\
& \leq M_{\theta}^{2} \sum_{n=1}^{\infty} \frac{1}{\left|\lambda_{\hat{k} n}\right|}\left|B^{*} \phi_{\hat{k} n}\right|^{2} \\
& <\infty \text { for any } t>0,
\end{aligned}
$$

since the domain of $\sqrt{-A_{\hat{k}}}$ is $V$ and $B \in L\left(\mathbf{R}, V^{\prime}\right)$. Next we have

$$
\begin{aligned}
\left(\sqrt{-A_{\hat{k}}}\right)^{-1} \dot{x}_{2}(t) & =-\sqrt{-A_{\hat{k}}} x_{2}(t) \\
& +\left(\sqrt{-A_{\hat{k}}}\right)^{-1} B g_{2}(t) .
\end{aligned}
$$

For the second term it holds that

$$
\begin{aligned}
& \left\|\left(\sqrt{-A_{\hat{k}}}\right)^{-1} B g_{2}(t)\right\|_{H} \\
& \leq\left\|\left(\sqrt{-A_{\hat{k}}}\right)^{-1} B\right\|_{H} \sup _{t \geq 0}\left|g_{2}(t)\right| \\
& \quad<\infty \text { for any } t>0 .
\end{aligned}
$$

From eqs.(22)and(23)we have

$$
\left\|\left(\sqrt{-A_{\hat{k}}}\right)^{-1} \dot{x}_{2}(t)\right\|<\infty \text { for any } t>0 \text {. }
$$

We have proved the lemma.

\section{Example}

In this section we give an example to illustrate our theory. Let us consider the following unstable heat system.

$$
\left\{\begin{array}{l}
x_{t}(t, z)=x_{z z}(t, z)+a x(t, z), a>0 \\
x(0, z)=x_{0}(z) \\
x_{z}(t, 0)=-u(t)-\theta_{1}-\theta_{2} \sin (t), x_{z}(t, 1)=0 \\
y(t)=\int_{0}^{1} c(z) x(t, z) d z=x(t, 0)
\end{array}\right.
$$

where $a$ is an unknown positive constant parameter, and $\theta_{1}, \theta_{2}$ are unknown constants. It can be shown that this system is equivalent to a Cauchy problem of eq. $(1)^{1)}$. For the system the operator 
$A$ is $d^{2} / d z^{2}+a I$ with obvious domain and $A$ has the eigenset

$$
\left\{\begin{array}{c}
\lambda_{n}=-(n-1)^{2} \pi^{2}+a \\
n=1,2, \cdots \\
\phi_{1}(z)=1, \phi_{n}(z)=\sqrt{2} \cos (n-1) \pi z \\
n=2,3, \cdots
\end{array}\right.
$$

The operator $A$ is self-adjoint and generates an analytic semigroup $S(t)$ on $H=L^{2}(0,1)$. The operator $B$ can be identified with the delta function $\delta(z)^{1)}$, and thus the actuator and sensor are collocated, that is, $C=B^{*}$. The system has unbounded $B$ and $C$ operators. One way of obtaining $V$ and $V^{\prime}$ spaces is to introduce the following weighted Hilbert space.

$$
V=\left\{x=\left.\sum_{n=1}^{\infty} x_{n} \phi_{n}\left|\sum_{n=1}^{\infty} \beta_{n}\right| x_{n}\right|^{2}<\infty\right\}
$$

with the inner product $\langle x, h\rangle_{V}=\sum_{n=1}^{\infty} \beta_{n} x_{n} h_{n}$, where $x_{n}=\left\langle x, \phi_{n}\right\rangle_{H}$ and

$$
\beta_{n}=\left\{\begin{array}{c}
1 \quad \text { if } n \in \Delta=\left\{n \in\{1,2, \cdots\} \mid \lambda_{n}>-1\right\} \\
\left|\lambda_{n}\right| \text { if } n \in \Gamma=\left\{n \in\{1,2, \cdots\} \mid \lambda_{n} \leq-1\right\} .
\end{array}\right.
$$

Then the dual space $V^{\prime}$ is defined by

$$
V^{\prime}=\left\{x=\left.\sum_{n=1}^{\infty} x_{n} \phi_{n}\left|\sum_{n=1}^{\infty} \frac{1}{\beta_{n}}\right| x_{n}\right|^{2}<\infty\right\}
$$

The coercivity condition holds as follows. For $x \in V$,

$$
\begin{aligned}
\langle-A x, x\rangle & =\sum_{n=1}^{\infty}\left(-\lambda_{n}\right) x_{n}^{2} \\
& =-\sum_{n \in \Delta} \lambda_{n} x_{n}^{2}+\sum_{n \in \Gamma} \beta_{n} x_{n}^{2} \\
& =\sum_{n=1}^{\infty} \beta_{n} x_{n}^{2}-\sum_{n \in \Delta}\left(\lambda_{n}+1\right) x_{n}^{2} \\
& \geq\|x\|_{V}^{2}-\max _{n \in \Delta}\left(\lambda_{n}+1\right) \sum_{n \in \Delta} x_{n}^{2} \\
& \geq\|x\|_{V}^{2}-\max _{n \in \Delta}\left(\lambda_{n}+1\right)\|x\|_{H}^{2} .
\end{aligned}
$$

Moreover it can be easily shown that $B \in$ $L\left(\mathbf{R}, V^{\prime}\right)$. Thus Assumption 1 holds. We investigate the eigenvalues of the operator $A-k B B^{*}$. This leads the following eigenvalue problem.

$$
\left\{\begin{array}{l}
x_{z z}(z)+a x(z)=\lambda x(z) \\
x_{z}(0)=k x(0), \quad x_{z}(1)=0
\end{array}\right.
$$

In this case the equation providing the eigenvalues is

$$
\sqrt{a-\lambda} \tan \sqrt{a-\lambda}=k .
$$

This equation has infinitely many zeros $\left\{\lambda_{k n}\right\}$ satisfying

$$
\begin{array}{r}
-\left(n-\frac{1}{2}\right)^{2} \pi^{2}+a<\lambda_{k n}<-(n-1)^{2} \pi^{2}+a \\
n=1,2, \cdots .
\end{array}
$$

Moreover it holds that

$$
\max _{n} \lambda_{k n}=\lambda_{k 1} \rightarrow a-\frac{\pi^{2}}{4}, \quad k \rightarrow \infty .
$$

Corresponding to the eigenvalues $\lambda_{k n}$ we have the complete orthonormal system of eigenfunctions

$$
\begin{aligned}
& \phi_{k n}(z)=\kappa_{n} \cos \left(\mu_{n}(1-z)\right), \quad n=1,2, \cdots \\
& \mu_{n}^{2}=-\lambda_{n} \\
& \kappa_{n}=\sqrt{\frac{2\left(k^{2}+\mu_{n}^{2}\right)}{k^{2}+\mu_{n}^{2}+k}}
\end{aligned}
$$

in $H=L^{2}(0,1)$. The eigenvalues $\lambda_{k n}$ are simple. Since the eigenvalues depend continuously on $\mathrm{k}$, there exists a positive constant $\hat{k}$ such that $\lambda_{\hat{k}}=\max _{n} \lambda_{\hat{k} n}<0$, if $a<\pi^{2} / 4$. Thus Assumption 2 holds. It should be noted that the transfer function of the system (25) is

$$
G(s)=\frac{\cosh \sqrt{s-a}}{\sqrt{s-a} \sinh \sqrt{s-a}}
$$

and the largest zero of $G(s)$ is a $-\pi^{2} / 4$.

We can construct an adaptive output-feedback stabilizer for the system of (25)

$$
\left\{\begin{array}{l}
u(t)=-k(t) y(t)-\hat{\theta}_{1}(t)-\hat{\theta}_{2}(t) \sin (t), \\
\dot{k}(t)=r y^{2}(t), \quad k(0)=0 \\
\dot{\hat{\theta}}_{1}(t)=\gamma_{1} y(t), \quad \hat{\theta}_{1}(0)=\hat{\theta}_{10}, \quad \gamma_{1}>0 \\
\dot{\hat{\theta}}_{2}(t)=\gamma_{2} \sin (t) y(t), \quad \hat{\theta}_{2}(0)=\hat{\theta}_{20}, \quad \gamma_{2}>0
\end{array}\right.
$$

if the unknown parameter $a<\pi^{2} / 4$.

We give a simulation result by eigenfunctions expansion in the case where $a=2, \theta_{1}=2, \theta_{2}=1$, $x_{0}(z)=1$. Fig. 1 shows plots of the state $x(t, z)$. Fig. 2 shows plots of the input $u(t)$, the gain $k(t)$, the parameters $\hat{\theta}_{1}(t)$ and $\hat{\theta}_{2}(t)$, respectively. In simulation we choose $\hat{\theta}_{10}=\hat{\theta}_{20}=0, \mathrm{r}=200, \gamma_{1}=15$, $\gamma_{2}=100$. These numerical results demonstrate the efficiency of the theory.

\section{Conclusion}

We have designed an adaptive regulator for some collocated distributed parameter systems with unbounded input and output operators in the case of bounded deterministic disturbances. The adaptive regulator has been constructed by the 


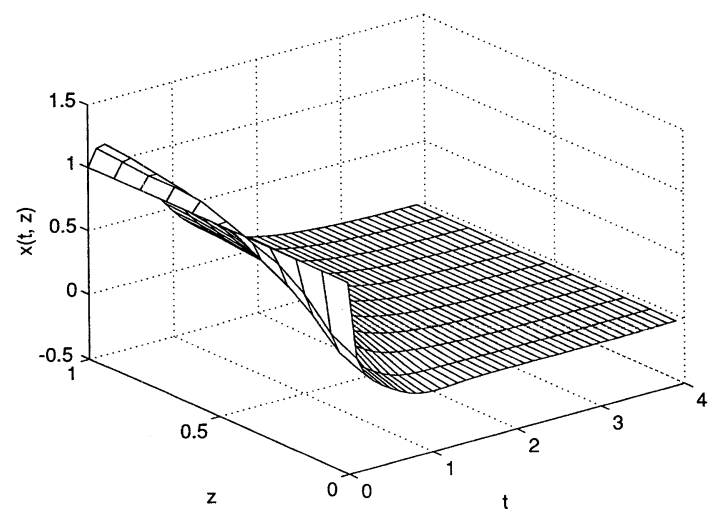

Fig. 1 Trajectory of $x(t, z)$
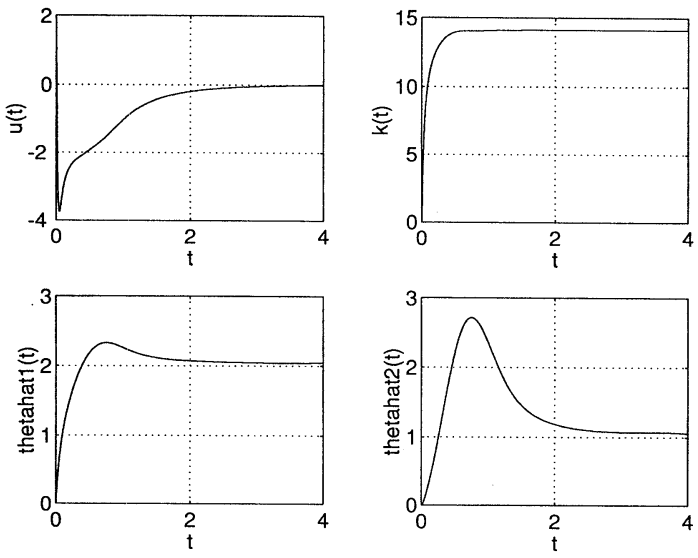

Fig. 2 Plots of $u(t), k(t), \hat{\theta}_{1}(t)$ and $\hat{\theta}_{2}(t)$

concept of high-gain output feedback and the estimation mechanism of the unknown parameters for the disturbance. In the control system, the convergence of the system state to zero is guaranteed. Lastly we should note that the self-adjointness condition of the operator A is not essential. We can directly extend our theory to the systems with non self-adjoint discrete spectral operator A.

\section{References}

1) R. F. Curtain and P. J. Pritchard: Infinitedimensional linear systems theory; Lecture Notes in Control and Information Sciences, No. 8, Springer (1978)

2) E. J. Davison: Multivariable tuning regulators: The feedforward and robust control of a general servomechanism problem; IEEE Trans. on Automatic Control, Vol. 21, No. 1, pp. 35-47 (1976)
3) K. S. Hong, J. W. Wen and K. I. Lee: New conditions for the exponential stability of evolution equations; IEEE Trans. on Automatic Control, Vol. 39, No. 7, pp. 1432-1436 (1994)

4) T. Kobayashi: Global adaptive stabilization of infinite dimensional systems; Systems and Control Letters, Vol. 9, No. 3, pp. 215-223 (1987)

5) T. Kobayashi: Zeros and design of contrlo systems for distributed parameter systems; Int. J. Systems Sciense, Vol. 23, No. 9, pp. 1509-1515 (1992)

6) J. L. Lions: Optimal Control of Systems Governed by Partial Differential Equations, Springer-Verlag (1971)

7) H. Logemann, and A. Ilchmann: An adaptive servomechanism for class of infinite-dimensional systems; SIAM. J. Control and Optimization, Vol. 32, No. 4, pp. 917-936 (1994)

8) H. Logemann, and B. Martensson: Adaptive stabilization of infinitr-dimenisional systems; IEEE Trans. on Automatic Control, Vol. 37, No. 12, pp. 1869-1883 (1992)

9) H. Logemann, and H. Zwart: Some remarks on adaptive stabilization of infinite-dimensional systems; Systems and Control Letters, Vol. 16, No. 3, pp. 199-207 (1991)

10) A. Pazy: Semigroups of Linear Operators and Applications to Partial Differential Equations, Springer-Verlag (1983)

11) S. Pohjolainen: Computation of transmisson zeros for distributed parameter systems; Int. J. Control, Vol. 33, No. 2, pp. 199-212 (1981)

12) S. Pohjolainen: Robust multivariable PI-contrpller for infinite dimensional systems; IEEE Trans. on Automatic Control, Vol. 27, No. 1, pp. 17-31 (1982)

13) H. Tanabe: Equations of Evolusion, Pitman (1979)

14) S. Townley: Simple adaptive stabilization of output feedback stabilizable distributed parameter systems; Dynamics and Control, Vol.5, pp. 107123 (1995) 
